\title{
Epileptic Seizures in Non-syndromic Megalencephaly (MEG): A Case Series in our Department
}

\author{
Federica Sullo ${ }^{1}$, Silvia Marino ${ }^{2}$, Milena Motta $^{1}$, Valeria Venti ${ }^{1}$, Stefania Zampogna ${ }^{3}$, Giulia Pecora ${ }^{1}$, \\ Andrea Praticò ${ }^{1}$, Francesca Patanè ${ }^{1}$, Giulia Salomone ${ }^{1}$, Bruna Scalia ${ }^{1}$, Luca Sabino ${ }^{1}$, Piero Pavone ${ }^{2 *}$ \\ ${ }^{1}$ Department of Clinical and Experimental Medicine, Section of Pediatrics and Child Neuropsychiatry, AUO Policlinico-Ove, University of \\ Catania, Italy \\ ${ }^{2}$ University-Hospital "Policlinico-Vittorio Emanuele," University of Catania, Italy \\ ${ }^{3}$ Hospital "PUGLIESE-CIACCIO" Catanzaro, Italy
}

*Corresponding author: Piero Pavone, University-Hospital “Policlinico-Vittorio Emanuele”, University of Catania, Italy

\section{ARTICLE INFO}

Received: 幽 December 06, 2019

Published: 蔧 December 13, 2019

Citation: Federica Sullo, Silvia Marino, Milena Motta, Valeria Venti, Stefania Zampogna, Giulia Pecora, Andrea Praticò, Francesca Patanè, Giulia Salomone, Bruna Scalia, Luca Sabino, Piero Pavone. Epileptic Seizures in Non-syndromic Megalencephaly (MEG): A Case Series in our Department. Biomed J Sci \& Tech Res 23(5)-2019. BJSTR. MS.ID.003965.

\section{ABSTRACT}

Macrocephaly or Megalencephaly are two synonyms that define the presence of a head circumference measurements two standard deviations above the age-related mean. Usually thee terms include patients characterized by large head associated with possible neurologic features, including neuro developmental impairment, both in motor and in intellective skills, showing intellective disability, together with possible occurrence of epileptic seizures. Despite this possible overlap between the two terms, is actually justified to distinguish the two eventualities due to different possible causal events, presence of specific cerebral anomalies, and clinical management. We selected a total of 10 patients ( 7 male, 3 female), observed in the period from July 2013 through July 2019, who were affected from non-syndromic MEG followed up at the pediatric and neuropediatric disorders center of Pediatric Unity of the University Hospital Policlinic-Vittorio Emanuele in Catania, Italy. 5 patients ( 4 males and 1 females) of the sample, presented the simultaneous presence of abnormally large head, mild/moderate developmental delay, and seizures.

Keywords: Epileptic Seizures; Megalencephaly; Macrocephaly; Large Head; Anatomic Megalencephaly

\section{Introduction}

With Megalencephaly (MEG) and macrocephaly are defined the presence of a head circumference measurements two standard deviations above the age-related mean [1,2]. Despite both these terms encompass individuals with a large head circumference, a distinction between megalencephaly and macrocephaly has been proposed due to the possible association with neurologic manifestations including intellective disability of various degree, epileptic seizures, and motor impairment. Since the wide heterogeneity in causative events, cerebral structural anomalies, approach in the work-up, treatment and prognosis for which a clinical distinction is justified [2,3]. Macrocephaly is referred to conditions in which the brain enlargement is a consequence of events inside the brain such as intracranial masses, hydrocephalus ex vacuo, abnormal ventricular dilatation and increase of bone skeletal structures. Whereas Megalencephaly refers to anomalous structural cerebral events such as ineffective molecular control of neuronal growth during the various stage of the brain development or to inborn errors of metabolism $[2,4]$.

The clinical conditions at the basis of MEG are quite uncommon, and can be distinct into three categories: idiopathic, metabolic, or anatomic. Especially Anatomic MEG may onset with neurologic signs, resulting isolated ("non-syndromic") or included in a syndromic phenotype, such as Megalencephaly-Capillary Malformation-Polymicrogyria (MCAP) and macrocephaly-cutis marmorata telangiectasica (M-CMTC) [4-6]. Nevertheless, there are examples where the adverse events causing megalencephaly and macrocephaly may co-exist but semantic distinction among these conditions is suitable. Our teamwork previously tried to 
highlight the clinical main features of MEG, focusing on the main disorders that manifest with this anomaly trying to categorize these disorders within the megalencephaly group [7]. In this paper we reviewed our patients with non-syndromic MEG, harboring minor dysmorphism, mild/moderate developmental delay and epileptic seizures as associated signs of the disorder: we enrolled 10 patients of MEG: five of them (unrelated patients) were affected by MEG and epilepsy.

\section{Materials and Methods}

From July 2013 through July 2019, 10 children with nonsyndromic MEG were selected (7 male, 3 female), and followed up at the Pediatric Unity of the University Hospital Policlinic-Vittorio Emanuele, Catania, Italy for pediatric and neuropediatric disorders; 5 of them (4 male, 1 female) have an abnormally large head, mild/ moderate developmental delay, and seizures.

\section{Editorial}

Among all the forms of MEG, the idiopathic form is the most frequent and is not associated with neurological manifestations, intellectual disabilities or other symptoms involving other parts of the body. In this disorder, the patient's head circumference increases gradually until an age of 18 months and then becomes more stable during development. The children's parental OFC measurements were within normal ranges. This situation is opposite to what is typically reported in the literature for benign MEG (i.e., a large parental head is frequently reported) [5]. Brain MRI malformative anomalies were are uncommonly reported in children with MEG. This situation confirms the hypothesis of Berg and Dobyns [4], who maintain that genetic anomalies affecting brain development in the first steps of neuronal growth may be the cause of cerebral involvement; structural cerebral anomalies my not always be noticeable in brain imaging techniques. This cohort of children exhibits a set of clinical signs consisting of an abnormally large head circumference (above the $97^{\text {th }}$ percentile), a mild-to-moderate intellectual disability, epileptic seizures in 5 cases and, in some cases, minor dysmorphism especially facial.

In the absence of signs suggestive of known syndromes, a diagnosis of anatomic, non-syndromic MEG was made. Nine of the ten patients did not show structural abnormality of the brain, in the group with epilepsy four of the five patients failed to exhibit

\section{ISSN: 2574-1241}

DOI: 10.26717/BJSTR.2019.23.003965

Piero Pavone. Biomed J Sci \& Tech Res

This work is licensed under Creative Commons Attribution 4.0 License

Submission Link: https://biomedres.us/submit-manuscript.php structural anomalies in their brain MRI. Only in one case we find a cerebral cortex dysplasia and micropolygyria. Among the children with epilepsy the EEG was abnormal and indicative of epilepsy diagnosis in all our patients; dysmorphisms were noted in three children; these conditions were not severe and mainly affected the face. A differential diagnosis between idiopathic MEG and anatomic, non-syndromic MEG is not simple prior to the onset of clinical signs. Developmental delays, although usually mild and moderate, and dysmorphisms, if not severe, may be useful for a diagnosis. We maintain that an abnormally large head deserves attention because this condition may hide relevant neurologic symptoms in the absence of evident brain anomalies. A large head in children with mild-to-moderate developmental delays and minor dysmorphisms may be a clue for subsequent epileptic seizures and a diagnosis of anatomic MEG.

\section{Acknowledgment}

We wish to thank the American Manuscript Editors (USA) for editing the manuscript.

\section{Statement of Ethics}

The authors have no ethical conflicts to disclose.

\section{Disclosure Statement}

The authors have no conflicts of interest to declare.

\section{References}

1. Sniderman A (2010) Abnormal head growth. Pediatr Rev 31(9): 382 384.

2. DeMyer W (1986) Megalencephaly: types, clinical syndromes, and management. Pediatr Neurol 2(6): 321-328.

3. Winden KD, Yuskaitis CJ, Poduri A (2015) Megalencephaly and Macrocephaly. Semin Neurol 35(3): 277-287.

4. Berg AT, Dobyns WB (2015) Progress in autism and related disorders of brain development. Lancet Neurology 14(11): 1069-1070.

5. Mroske C, Rasmussen K, Shinde DN, Huether R, Powis Z, et al. (2015) Germline activating MTOR mutation arising through gonadal mosaicism in two brothers with megalencephaly and neurodevelopmental abnormalities. BMC Med Genet 16: 102.

6. Strauss KA, Puffenberger EG, Robinson DL, Morton DH (2003) Type I glutaric aciduria, part 1: Natural history of 77 patients. Am J Med Genet C Semin Med Genet 121C (1): 38-52.

7. Pavone P, Praticò AD, Rizzo R, Corsello G, Ruggieri M, et al. (2017) A clinical review on megalencephaly: A large brain as a possible sign of cerebral impairment. Medicine (Baltimore) 96(26): e6814.

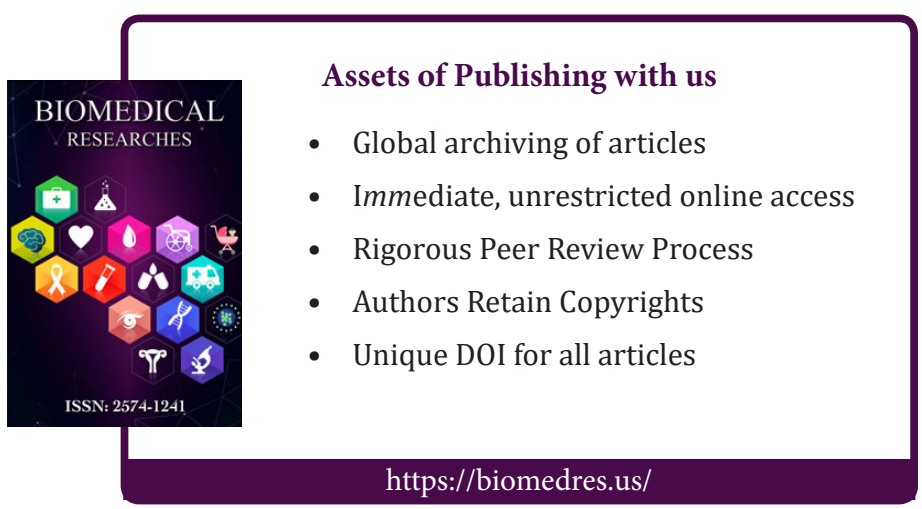

